equal to the normal upon the corresponding principal directrix plane. And the normal to the surface at the point bisects the exterior angle of the two lines.

In an hyperbolic paraboloid, for any point on the surface, the difference between the focal distances to the foci of the two focal parabolas is a constant. And the normal to the surface at the point bisects the exterior angle of the two lines.

The reader of this book will hope with Professor Staude " that the focal properties of the conicoid will attain the same recognition that the corresponding properties in the plane have long since enjoyed."'

PRINCETON,

H. D. Thompson.

February 25, 1898.

\title{
NOTE ON THE THEORY OF CONTINUOUS GROUPS.
}

Is a "Note on the fundamental theorems of Lie's theory of eontinuous groups," contributed to the BuLLETIN in November, 1897, by Dr. Lovett, attention is drawn to an error or misapprehension in a paper which I had the honor of contributing to the Proceedings of the Lonion Mathematical Society (vol. 23, p. 381-390).

The theorem to which objection is taken is: " If $x_{1}^{\prime}, \ldots, x_{n}$ ' is a point obtained from the point $x_{1}, \ldots, x_{n}$ by the operation

$$
1+X+\frac{X^{2}}{2 !}+\cdots
$$

and $x_{1}{ }^{\prime \prime}, \ldots, x_{n}{ }^{\prime \prime}$ is a point obtained from the point $x_{1}{ }^{\prime}, \ldots, x_{n}{ }^{\prime}$ by the operation

$$
1+Y^{\prime}+\frac{Y^{\prime 2}}{2 !}+\cdots
$$

where

$$
X \equiv \lambda_{1} X_{1}+\cdots+\lambda_{r} X_{r}
$$

and

$$
Y \equiv \mu_{1} X_{1}+\cdots+\mu_{r} X_{r} \text {, }
$$

$X_{k}$ denoting the linear operator

$$
\sum_{i=1}^{i=n} \xi_{k i}\left(x_{1}, \cdots, x_{n}\right) \frac{\partial}{\partial x_{i}} ;
$$

then $x_{1}^{\prime \prime}, \ldots, x_{n}^{\prime \prime}$ can be directly derived from the point $x_{1}, \cdots, x_{n}$ by the operation 


$$
1+Z+\frac{Z^{2}}{2 !}+\cdots
$$

where

$$
Z \equiv \nu_{1} X_{1}+\cdots+\nu_{r} X_{r}
$$

provided that, for all values of $k, j$

$$
X_{k} X_{j}-X_{j} X_{k} \equiv \sum_{s=1}^{s=r} C_{k j s} X_{s}
$$

where $\lambda, \mu, \nu$, and $C$ are any functions of the variables."'

The ground of the objection is that I implicitly assume a theorem which Lie has only proved when $C_{k j s}$ is a constant. I wish to draw attention to the fact that in $\mathrm{my}$ proof I merely assume the obvious theorem

$$
\operatorname{Limit}_{n=\infty}\left(1+\frac{Y}{n}\right)^{n}=1+Y+\frac{Y^{2}}{2 !}+\cdots
$$

where $Y$ is any operator which obeys the distributive law. Lie's coustants $C_{k j s}$ cannot, of course, appear in a simple group ; and the fact that $Y \equiv \mu_{1} X_{1}+\cdots+\mu_{r} X_{r}$ cannot alter the fact that $1+Y+\frac{Y^{2}}{2 !}+\cdots$ is a simple group, though it can represent an infinity of simple groups by varying the forms of the functions $\mu_{1}, \cdots, \mu_{r}$.

It is not asserted that, if

$$
X_{k} X_{j}-X_{j} X_{k} \equiv \sum_{s=1}^{s=r} C_{k j s} X_{s}
$$

then the operators $X_{1}, \cdots, X_{r}$ generate a finite continuous group but they do generate a continuous group. [I fear this is not clearly expressed in my paper.] Finally, there is not, so far as I can see, any discrepancy between the result which I have given and Lie's theorems; the latter are only concerned with finite continuous groups, though the reasoning by which they are established would, I think, hold good equally if his parameters $a_{1}, \cdots, a_{r}$ instead of being taken as constants were taken as arbitrary functions of $x_{1}, \cdots, x_{n}$, and the infinitesimal transformations formed by considering the effect of a small change in the form of any one of the functions. The conclusions would then, however, be nugatory unless for intransitive groups for which $r>n$.

J. E. Campbell.

Hertford College, OXford. 